CHAPTER 6

\title{
The Gaseous Cosmic Web
}




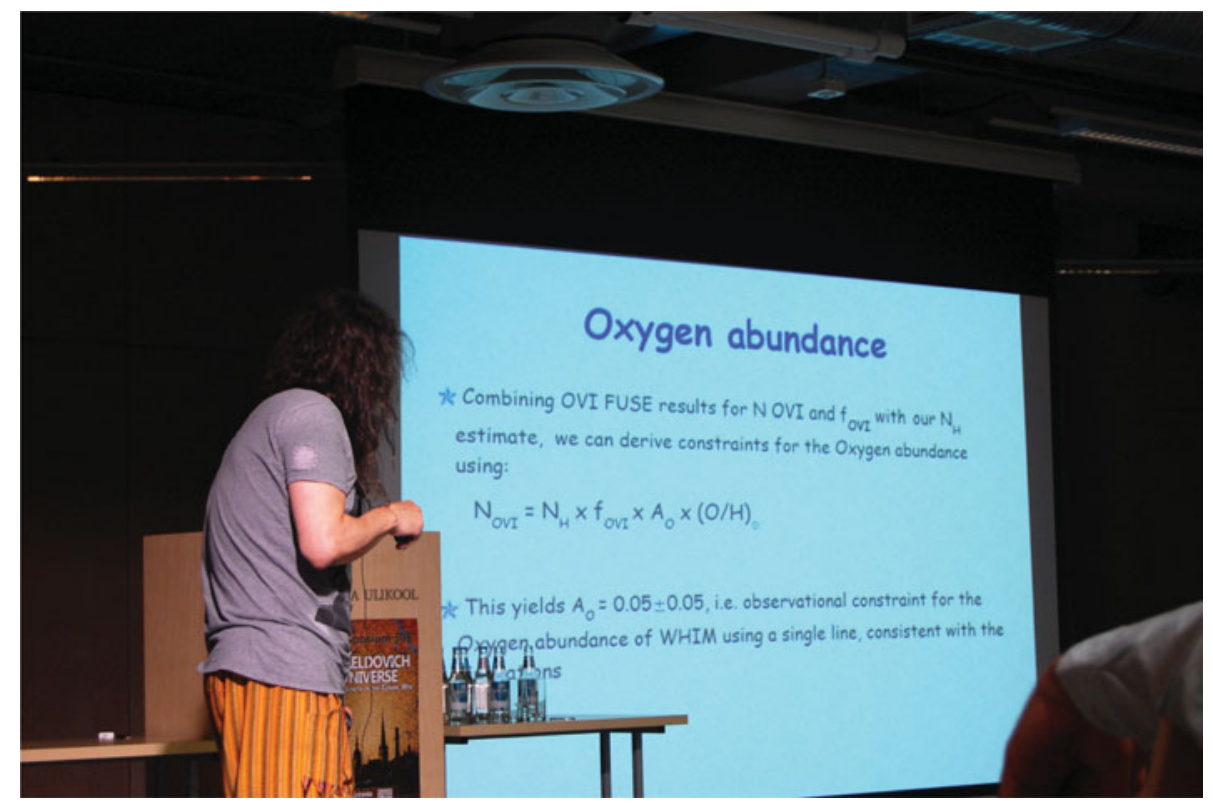

Jukka Nevalainen absorbed by WHIM.

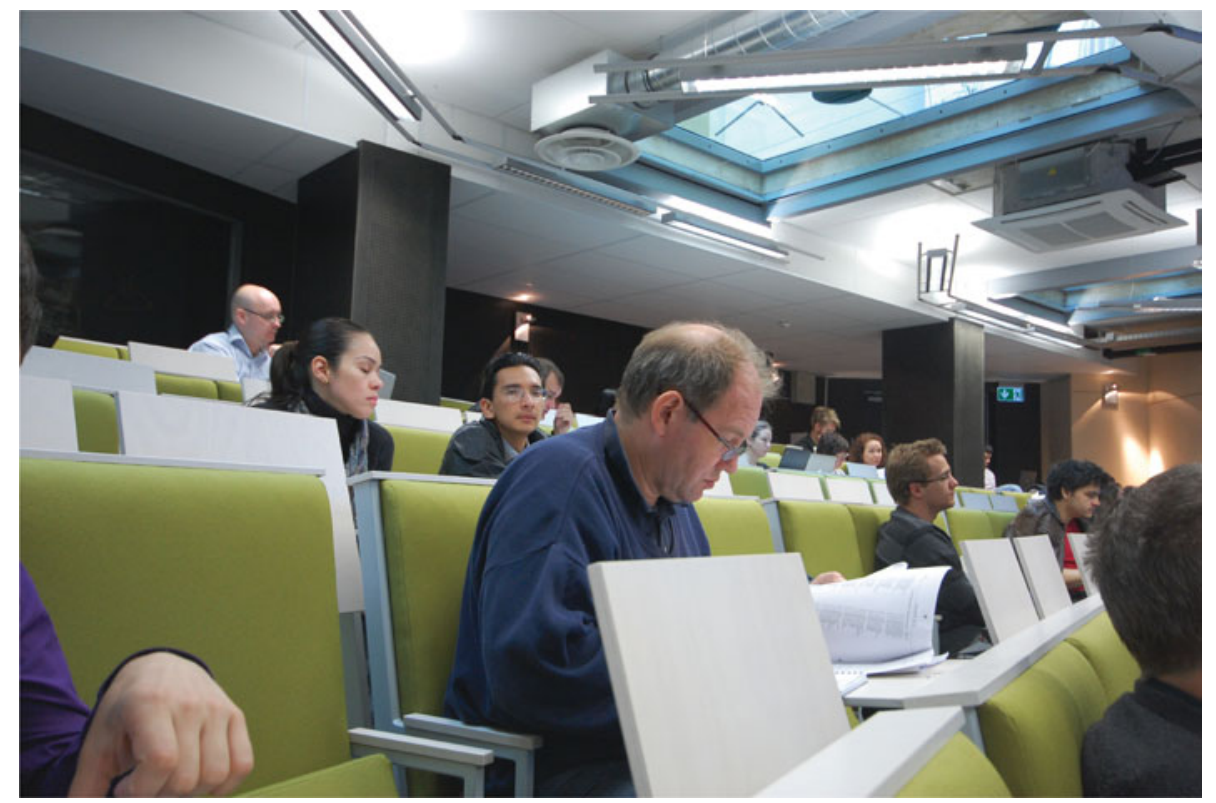

Per Lilje in a serious assessment of presented matters. 


\title{
The Ly $\alpha$ forest and the Cosmic Web
}

\author{
Avery Meiksin \\ Institute for Astronomy, University of Edinburgh, \\ Edinburgh, Scotland, U.K. \\ email: a.meiksin@ed.ac.uk
}

\begin{abstract}
The accurate description of the properties of the Lyman- $\alpha$ forest is a spectacular success of the Cold Dark Matter theory of cosmological structure formation. After a brief review of early models, it is shown how numerical simulations have demonstrated the Lyman- $\alpha$ forest emerges from the cosmic web in the quasi-linear regime of overdensity. The quasi-linear nature of the structures allows accurate modeling, providing constraints on cosmological models over a unique range of scales and enabling the Lyman- $\alpha$ forest to serve as a bridge to the more complex problem of galaxy formation.
\end{abstract}

Keywords. intergalactic medium, quasars: absorption lines, galaxies: formation, large-scale structure of universe, dark matter, cosmological parameters

\section{Introduction}

One of the great achievements of twentieth century cosmology is the development of the Cold Dark Matter (CDM) paradigm. It provides a framework for describing the growth of large-scale cosmological structure formation from galactic scales to the cosmological horizon. Within the context of Friedmann-Lemaître-Robertson-Walker expanding universe models, the precise predictions of $\Lambda \mathrm{CDM}$ for the angular structure of fluctuations in the Cosmic Microwave Background (CMB) have been confirmed with an accuracy previously unprecedented in cosmology. It provides a broadly successful description of the abundance of galaxies and their clustering, and will be a key ingredient in a complete description of the origin of galaxies.

One of the major successes of the $\Lambda \mathrm{CDM}$ model is its description of the gaseous material between the galaxies, the Intergalactic Medium (IGM). The accuracy of its predictions for the IGM are second only to the CMB, and are of even wider phenomenological scope. With only one principal adjustable parameter, the mean ionization background, the statistics of the neutral hydrogen component are recovered with spectacular success.

The intergalactic medium acts also as the arena for galactic and Active Galactic Nuclei (AGN) feedback and provides a proving ground for theories of galaxy formation. In addition to hydrogen and helium, the IGM contains metals in various ionization stages. These are believed to have been deposited by galaxies through wind ejection during energetic periods of star formation. The enhanced ionization near Quasi-Stellar Objects (QSOs) (the "proximity effect") allows estimates of the Ultra-Violet (UV) metagalactic ionizing background which agree with the values required by simulations. The levels suggest QSOs contribute substantially to the ionizing budget, and may dominate at redshifts $1<z<3$, but are not adequate alone at higher redshifts. The most likely candidates for additional ionizing photons are galaxies, yet the direct detection of the required ionizing flux continues to prove elusive. The helium was almost certainly ionized by the hard radiation from QSOs. The ionization scenario itself, however, is still uncertain, and may have been protracted in time. At still earlier times, the ionization impact of the first stars 


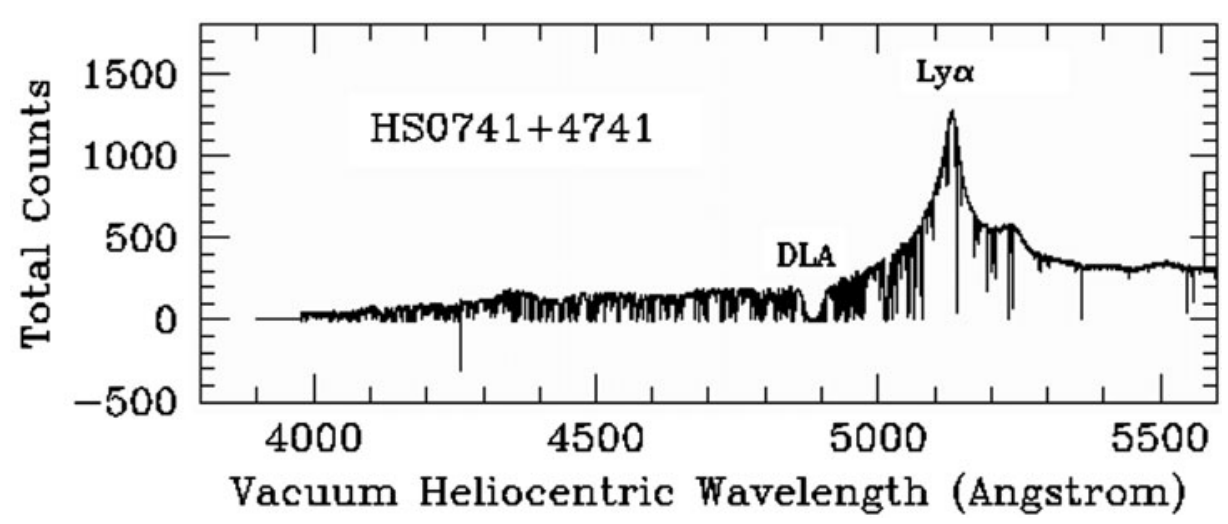

Figure 1. Spectrum of the $z=3.2$ QSO HS0741+4741. The redshifted Ly $\alpha \lambda 1216 \mathrm{~A}$ emission line of the QSO is indicated. The fluctuations at shorter wavelengths comprise the Ly $\alpha$ forest, including a prominent Damped Ly $\alpha$ Absorption (DLA) system. Absorption features at longer wavelength arise from intervening absorption by metal ions such as C II, C IV, Si IV (Songaila 1996). (Figure courtesy of A. Songaila.)

will produce a distinctive signature in the $21 \mathrm{~cm}$ absorption from the IGM against the CMB during the Epoch of Reionization.

Most recently, the study of the IGM has been undergoing a renascence with tomographic observations made possible by the huge numbers of bright QSOs delivered through ever deeper sky surveys. No longer are studies restricted to the occasional lineof-sight to a background QSO: multiple QSOs nearby on the sky are making 3D tomographic probing a reality. Motivated by attempts to constrain the evolution of the "dark energy" equation of state, surveys with multiple lines of sight passing near foreground galaxies are also fast presenting a novel means for probing the environment of the galaxies, including the possibility of the direct detection of the impact of galactic winds.

A comprehensive review of the observations and physics of the intergalactic medium is provided in Meiksin (2009).

\section{Observational probes of the IGM}

The main observational phenomenon for testing model predictions of the IGM is the Ly $\alpha$ forest, the absorption features visible in the spectra of high redshift Quasi-Stellar Objects (QSOs) due to the scattering of Ly $\alpha$ photons by intervening intergalactic neutral hydrogen. A representative spectrum is shown in Fig. 1. The redshifted Ly $\alpha \lambda 1216 \mathrm{~A}$ emission line is clearly visible. The "noise" at shorter wavelengths is not noise at all, but the Ly $\alpha$ forest. For comparison, the noise level is apparent at longer wavelengths, where even there absorption lines are visible. Some of these are also due to intervening intergalactic gas, but from ions of metals, such as carbon and silicon, mixed in the gas.

The flux fluctuations are characterized by their optical depths, which depend on the $\mathrm{H}$ I column density $N_{\mathrm{HI}}$ and velocity width, or Doppler parameter $b$, of the system:

$$
\tau_{0}^{\mathrm{HI}} \simeq 0.38\left(\frac{N_{\mathrm{HI}}}{10^{13} \mathrm{~cm}^{-2}}\right)\left(\frac{b}{20 \mathrm{~km} \mathrm{~s}^{-1}}\right)^{-1} .
$$

The absorption systems are classified according to the inferred $\mathrm{H}$ I column densities: systems with $\log _{10} N_{\mathrm{HI}}>17.2$ are Lyman Limit Systems, as they are optically thick at the $\mathrm{H}$ I photoelectric edge, while systems with $\log _{10} N_{\mathrm{HI}}>20.3$ are Damped Ly $\alpha$ absorbers, showing prominent radiation damping wings from predominantly neutral hydrogen. The 
Table 1. Summary of absorption line system properties

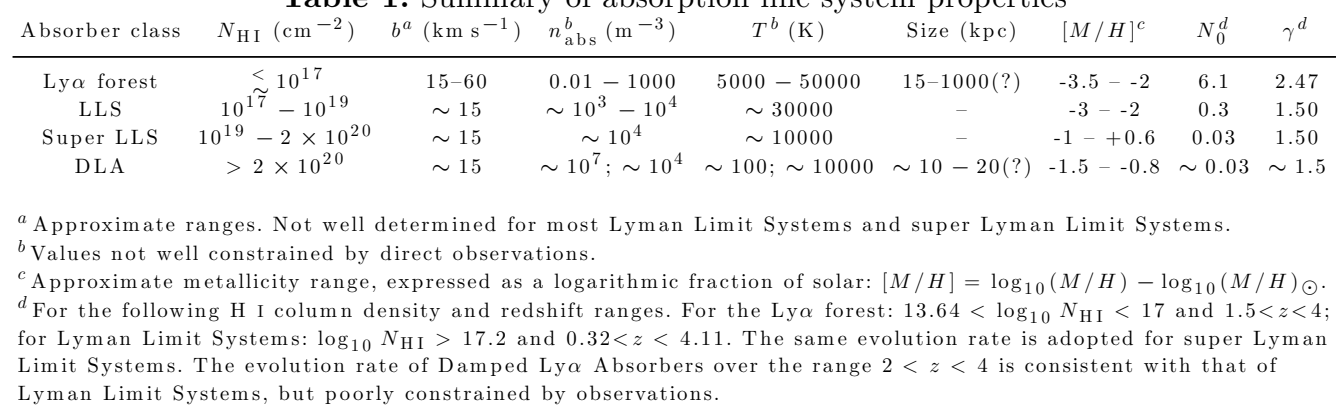

number of systems per unit redshift interval evolves rapidly, approximately as a power law $d N / d z=N_{0}(1+z)^{\gamma}$. Characteristic values for the absorbers and their inferred physical properties are provided in Table 1 .

The absorption systems contribute substantially to the effective optical depth of the IGM, defined through $\exp \left(-\tau_{\text {eff }}\right)=\langle\exp (-\tau)\rangle$, where the average is carried over a broad region of a spectrum with flux values $\exp (-\tau)$, where $\tau$ denotes the optical depth per pixel. For absorption systems optically thin at line centre,

$$
\tau_{\mathrm{eff}} \simeq \frac{3}{8 \pi} \Gamma_{\alpha} \lambda_{\alpha}^{3} \frac{\left\langle n_{1}\right\rangle}{H(z)}
$$

where $\Gamma_{\alpha}$ is the spontaneous decay rate of the Ly $\alpha$ transition, with line-centre wavelength $\lambda_{\alpha},\left\langle n_{1}\right\rangle$ is the spatially averaged density of neutral hydrogen and $H(z)$ is the Hubble parameter at redshift $z$. In the limit that the hydrogen is uniform, the expression is known as the Gunn-Peterson optical depth (Gunn \& Peterson 1965). For a set of discrete absorption systems of internal hydrogen density $n_{\text {abs }}$, the spatially averaged hydrogen density is $\left\langle n_{1}\right\rangle=Q_{\mathrm{abs}} n_{\mathrm{abs}}$, where $Q_{\mathrm{abs}}$ is the volume filling factor of the clouds.

If the gas density in the absorbers is so high the absorption features become optically thick, then the effective optical depth reduces to

$$
\tau_{\text {eff }} \simeq 3 Q_{\text {abs }} \frac{b}{H(z) L},
$$

where $b$ is the velocity width of the absorbers and $L$ is their line-of-sight thickness. For typical values of $b=25 \mathrm{~km} \mathrm{~s}^{-1}$ and $L=15 \mathrm{kpc}$ from Table 1 , an optical depth of $\tau_{\text {eff }} \lesssim 0.3$ at $z=3$ would correspond to $Q_{\mathrm{abs}} \simeq 0.02$, so that optically thick absorbers would occupy only a small volume of the IGM.

The possibility of a dense underbrush of unresolved optically thin Ly $\alpha$ lines frustrated any attempt to secure the discovery of a diffuse homogeneous component of the IGM (e.g., Jenkins \& Ostriker 1991). At the same time, size constraints on the absorbers from common features measured in parallel lines of sight gave preference to large systems tens of kiloparsecs across (e.g., Smette et al. 1992), although the errors were large. A possible way out of the absence of any Gunn-Peterson signal was that a large fraction, possibly most, of the baryons were contained in the Ly $\alpha$ forest, leaving only a small residual diffuse component (McGill 1990; Meiksin \& Madau 1993), although such a conclusion depended on the unknown geometry of the absorbers (Rauch \& Haehnelt 1995).

\section{Early models}

The simplest models for the systems giving rise to the Ly $\alpha$ absorbers were isolated gaseous clouds, probably motivated by the analogy of nebular clouds in the interstellar 
medium of the Milky Way. If the IGM were neutral, the spectrum of a QSO source shortward of its Ly $\alpha$ emission line would be black, so great is the resonance line scattering cross section for Ly $\alpha$. The absence of a detectable trough led to the suggestion that the IGM was most likely highly ionized (Gunn \& Peterson 1965). A model put forward for the Ly $\alpha$ absorbers was one of clouds pressure-confined by a hot ionized IGM (Sargent et al. 1980). The origin of the clouds was unexplained. In succeeding years, suggestions included supernova remnants in galactic haloes, winds from dwarf galaxies and shells of cosmologically expanding shock waves.

The Cold Dark Matter model of structure formation (Peebles 1982; Blumenthal et al. 1984; Peebles 1984) provided a natural origin: gas gravitationally bound to small dark matter haloes, so-called "minihaloes" (Rees 1986). A halo with a mass of $\sim 10^{9} M_{\odot}$ would retain gas at photoionization temperatures, and copious numbers of such haloes should exist at high redshifts. The Ly $\alpha$ forest would have been a triumphant prediction of the CDM model had it not already been observed.

The model generalizes: minihaloes are only one form of non-linear dark matter structures. Zel'dovich pointed out that gravitational collapse will generally produce 1D sheets (Zel'dovich 1970). Let $\mathbf{x}$ be the comoving position of a particle with initial comoving position q. Its peculiar velocity is then $\mathbf{v}=a \dot{\mathbf{x}}$, where $\Psi(\mathbf{q})$ describes the initial deformation of the density field, and $a(t)=1 /(1+z)$ is the expansion factor of the Universe at the epoch corresponding to redshift $z$. Zel'dovich showed that the positions of the particle coordinates will evolve approximately according to

$$
\mathbf{x}(\mathbf{q}, t)=\mathbf{q}-D(t) \nabla \Psi(\mathbf{q}), \quad \mathbf{v}(\mathbf{q}, t)=-a \dot{D}(t) \nabla \Psi(\mathbf{q}),
$$

where the gradient is with respect to $\mathbf{q}$ and $D(t)$ is the growth factor for linear perturbations, $D(t)=(\dot{a} / a) \int^{a} d a / \dot{a}^{3}$ (Peebles 1993).

The physical radius is related to the comoving radius through $\mathbf{r}=a(t) \mathbf{x}$. Conservation of mass gives for the density, up until the time of caustic formation, $\rho(\mathbf{x}, t)=$ $\rho(\mathbf{q}) /\left|d^{3} \mathbf{x} / d^{3} \mathbf{q}\right|$, where the denominator is the determinant of the Jacobian of the coordinate transformation. The solution is exact for a $1 \mathrm{D}$ slab. The density prior to caustic formation grows as $\rho(x, t)=\rho_{0} a(t)^{-3} /\left[1-D(t) d^{2} \Psi / d q^{2}\right]$. A density caustic forms when the denominator vanishes. Gravitational instability ensures that a uniform density ellipsoidal density perturbation will collapse most rapidly along its shortest axis, forming a "Zel'dovich pancake."

Ly $\alpha$ absorption systems would arise naturally as sheet-like caustics in physical space (Miralda-Escudé \& Rees 1993; Meiksin 1994) or even redshift space (McGill 1990). A wide range of models could then account for the Ly $\alpha$ forest, from spheres to sheets, from explosion-driven shocks to gravitational collapse.

Gathered in the city of Tallinn, with its medieval old city and thirteenth century monastery, we're reminded of the wisdom of the thirteenth century English monk William of Ockham and the point of logic he famously advocated on the economy of causes, known today as Occam's Razor: "Plurality should not be posited without necessity." The various models for the Ly $\alpha$ absorbers led to much discussion and debate as to which was the correct one. But another medieval doctrine rivaled Occam's Razor, dubbed by the historian A.O. Lovejoy the Principle of Plenitude: "The universe is a plenum formarum in which the range of conceivable diversity of kinds of ... things is exhaustively exemplified." In a phenomenologically rich subject like astrophysics, a wide range of causes often produce similar signals. Under the $\Lambda \mathrm{CDM}$ paradigm, the physical systems that give rise to the Ly $\alpha$ absorbers are indeed highly diverse. Occam's Razor instead prevails in another guise: the variation in structure of the absorbers is a consequence of the stochastic 

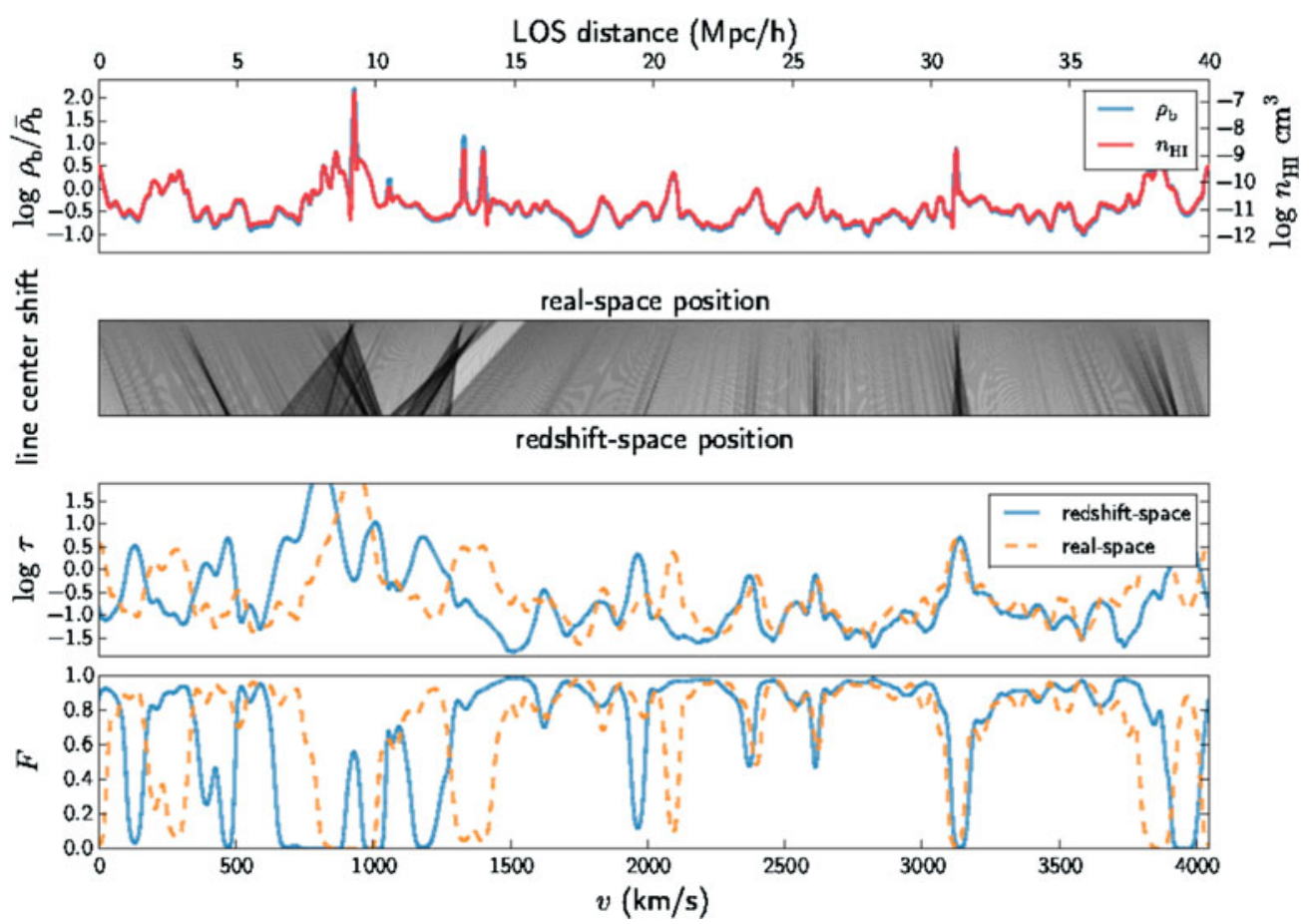

Figure 2. A line-of-sight cut through a $\Lambda$ CDM simulation with $2048^{3}$ grid zones in a box $40 h^{-1}$ Mpc (comoving) on a side of the Ly $\alpha$ forest at $z=2.5$. The top panel shows the total baryon overdensity and neutral hydrogen density, which are strongly correlated. The second panel shows the mapping between the real space and redshift space of gas parcels, allowing for the peculiar motion of the gas. The bottom two panels show the optical depth and flux, respectively, in both real space and velocity space. Redshift-space distortions both shift the positions of absorption features and alter their blending. From Lukić et al. (2014). (Figure reproduced by permission of the AAS.)

geometry of a 3D gaussian random field as realized in the process of large-scale structure formation (Bond et al. 1986; Bond, Kofman \& Pogosyan 1996).

\section{Cosmological simulations}

By the late 1980s, a paradigm shift was underway in the description of the origin and structure of the Ly $\alpha$ forest: the absorption features would arise from the filamentary structures seen in cosmological hydrodynamical simulations (Lattanzio, Bond \& Monaghan 1989). A flurry of simulations followed, using both grid-based (Cen et al. 1994; Zhang et al. 1995; Miralda-Escudé et al. 1996) and particle-based (Katz et al. 1996; Wadsley and Bond 1997) hydrodynamics codes. The results were sensational. Exquisite agreement was achieved between the simulations and observations of the Ly $\alpha$ forest.

Lines of sight through a simulation volume reveal that features originate from a range of moderate density fluctuations, and from underdense regions as well, as shown in Fig. 2. Underlying thermal motions will broaden spectral features relative to the density fluctuations, while bulk motions shift the features, sometimes sharpening them into structures similar to velocity caustics.

The evolution of the numbers of absorbers is explained predominantly as a consequence of cosmological expansion. As the density of the Universe decreases with time, 

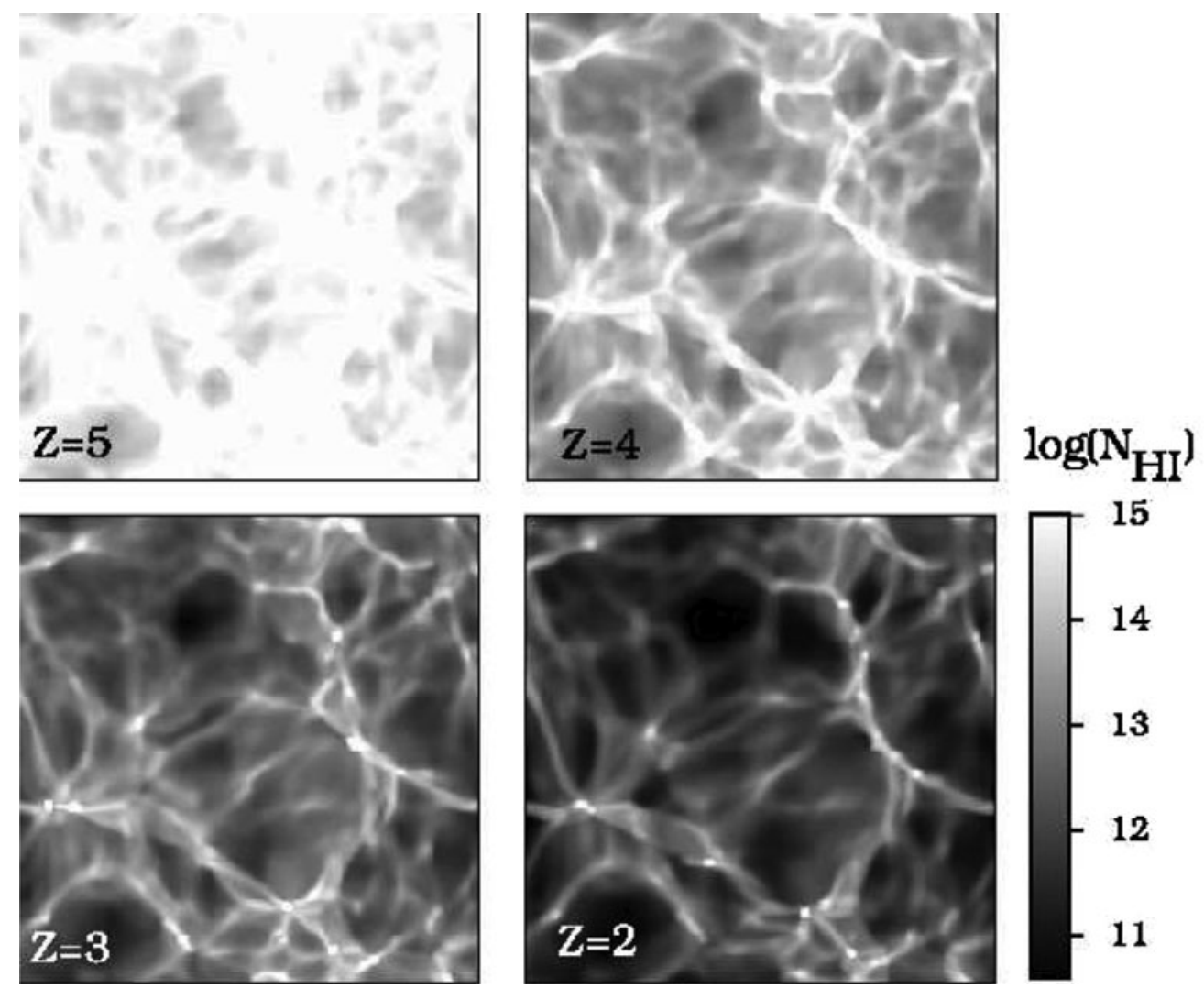

Figure 3. Evolution of the $\mathrm{H}$ I column density. The filamentary structure of the IGM is nearly stationary in the comoving frame. By contrast, the decline in the physical gas density results in rapid evolution of the $\mathrm{H}$ I column density, and the IGM becomes increasingly transparent with time. From Zhang et al. (1998). (Figure reproduced by permission of the AAS.)

the structures along a line of sight that give rise to systems of a given $\mathrm{H}$ I column density range become fewer and fewer, as shown in Fig. 3.

Fig. 4 shows that the absorption systems originate from systems with a variety of geometries, depending on column density. The highest column density systems originate in spheroidal haloes, which form at the intersections of elongated filamentary structures that give rise to the most abundant absorbers detected. Lower column density systems originate in sheets extending between the filaments, while the lowest column density systems, too small to detect in $\mathrm{H}$ I but visible as He II absorbers, derive from fluctuations in underdense regions.

The simulations produce excellent agreement with the statistical properties of the Ly $\alpha$ forest. One of the most basic is the distribution of flux per pixel. Agreement is acheived at the few percent level, as shown in Fig. 5. The data are sufficiently precise that this statistic has been used to discriminate between rival cosmologies and CDM power spectrum parameters (e.g., Meiksin et al. 2001).

The next higher order statistic, the flux power spectrum, shows excellent agreement at small wavenumbers (Croft et al. 1998). Following the measurement of the flux power spectrum using 3035 QSO spectra taken as part of the Sloan Digital Sky Survey (SDSS), cosmological simulations of the IGM were used to place constraints on the amplitude 


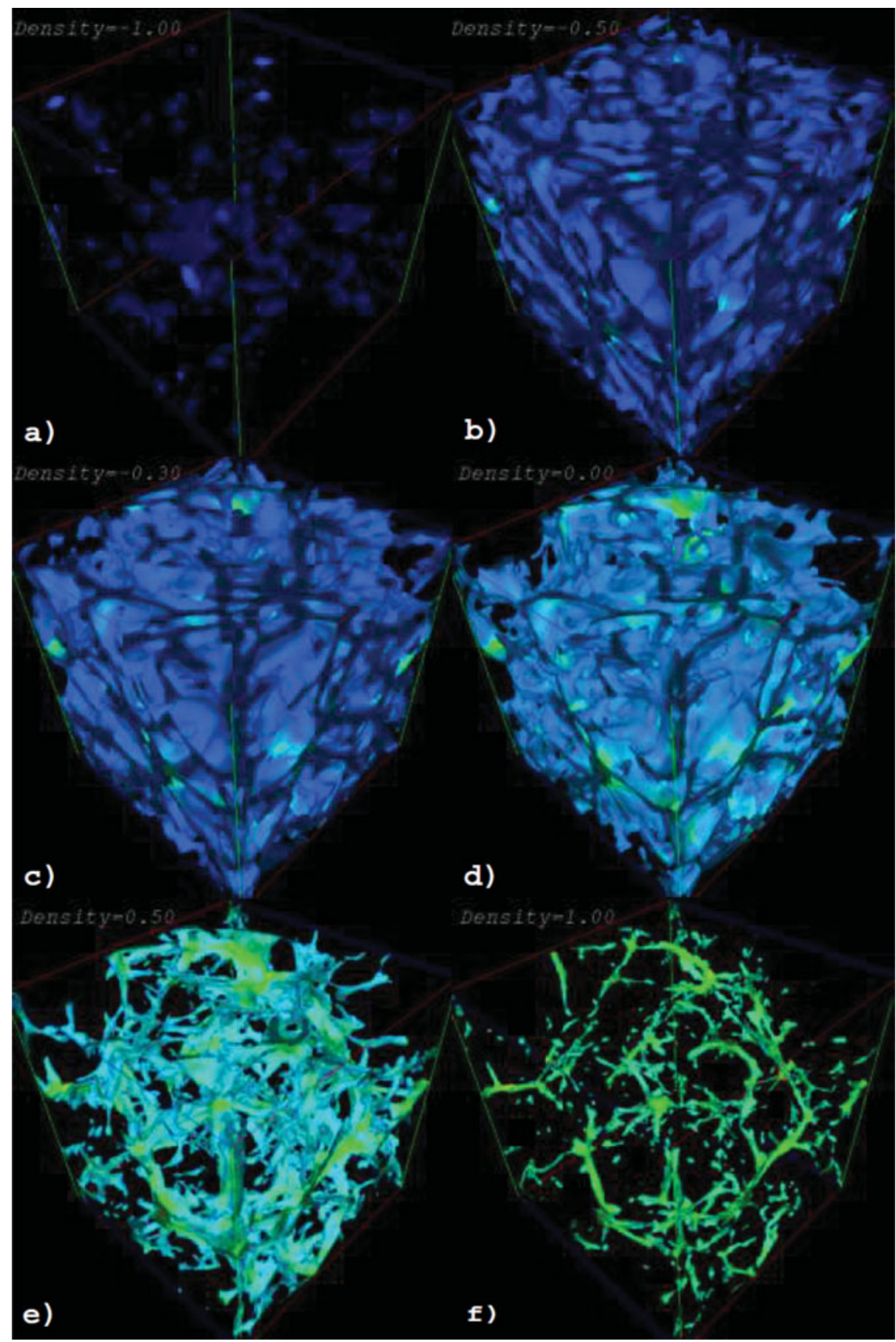

Figure 4. Isodensity contour surfaces of $\log$ baryon overdensity at $z=3$ for a flat Cold Dark Matter dominated universe. The contour levels are $\log _{10}(\rho /\langle\rho\rangle)=-1.0,-0.5,-0.3,0.0,0.5$ and 1.0. Low density regions are amorphous structures filling sheets at the mean density which intersect at overdense filaments. The filaments intersect at highly overdense collapsed halos. From Zhang et al. (1998). (Figure reproduced by permission of the AAS.) 


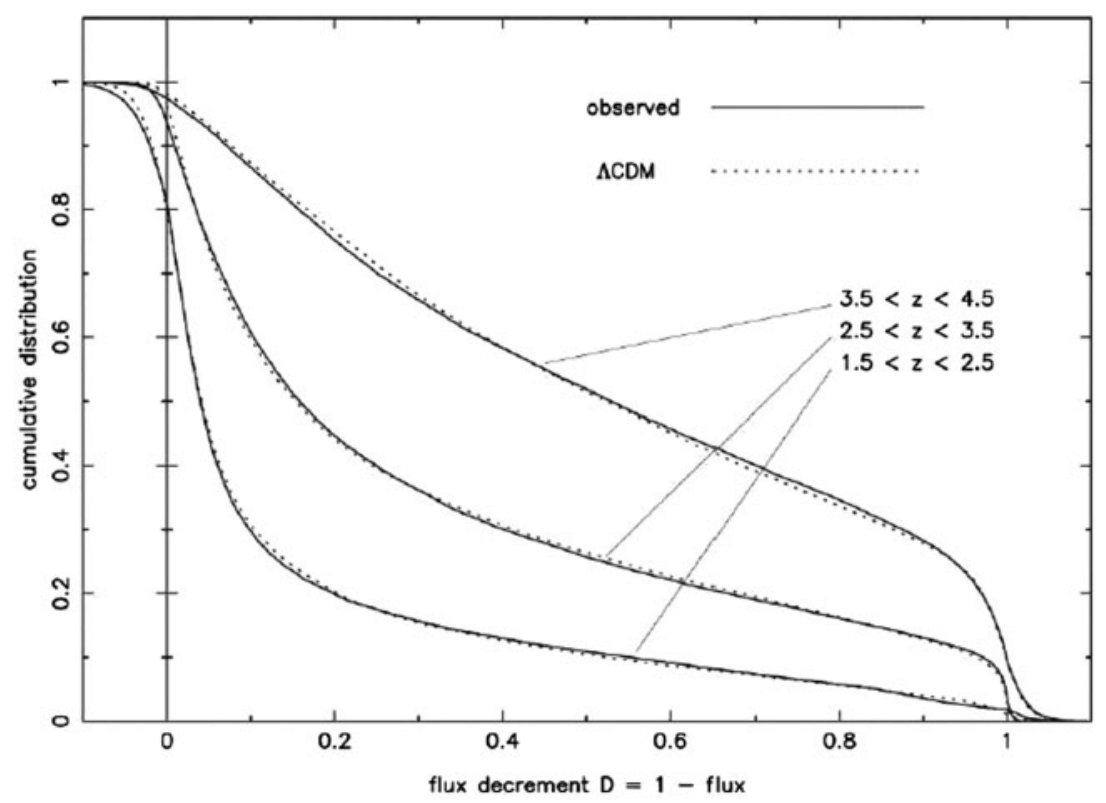

Figure 5. Comparison of the cumulative flux per pixel distribution from a $\Lambda$ CDM simulation with data based on high resolution Keck spectra of 7 QSOs. The data have been grouped into three redshift intervals and the observations corrected to take into account evolution in the mean transmission within each interval. Noise has been added to the simulated data to match the noise properties of the measured spectra. From Rauch et al. (1997). (Figure reproduced by permission of the AAS.)

and tilt of the primordial power spectrum (McDonald et al. 2005, Viel \& Haehnelt 2006) and on the summed mass of massive neutrino species (Viel, Haehnelt \& Springel 2010).

The predicted flux power spectra do not match the observations at high wavenumbers, where line crowding and broadening cut off the power. This is likely in part due to the uncertain temperature structure of the IGM. Early numerical simulations already revealed one important discrepancy between the simulations and the observations: the simulations predict a greater number of narrow lines than measured (Davé et al. 1997). The likely culprit is helium and its photoionization, which were not adequately treated.

Since the discovery of intergalactic helium (Jakobsen et al. 1994), the observational measurement campaign of intergalactic helium has raised many puzzles. The effective optical depth of singly-ionized helium fluctuates wildly above a redshift of $\sim 2.7$ (e.g., Syphers \& Shull 2014; Worseck et al. 2014). It is unknown why. It may indicate a fluctuating metagalactic UV background, as may be expected from the relatively rare QSOs, as only QSOs have an adequately hard spectrum to photoionize $\mathrm{He}+$; stellar-dominated galaxy spectra are too soft. Possibly the reionization of helium is a long and drawn out process. Since helium reionization will act as an extra source of heating, contributing to the thermal broadening of the lines, a theory of the structure of the IGM will remain incomplete without an understanding of the helium reionization process.

The reioniation of helium is one of several outstanding problems of the structure of the IGM. Another is the effect galaxy formation and feedback from forming stars and black holes have on the IGM, especially near galaxies. Indeed, simulations of the IGM offer the possibility of turning the problem around by using them to constrain galaxy formation models. 


\section{New directions}

Observational studies of the IGM continue to flourish, stimulating ever more accurate and realistic simulations. I conclude with some directions the field is taking or may take in the near future.

IGM tomography Motivated by using the Ly $\alpha$ forest to search for evolution in the dark energy equation of state, the BOSS experiment of SDSS-III has substantially increased the sky density of QSO lines of sight. The close proximity of large numbers of lines of sight has enabled 3D correlations in the forest to be measured over large scales for the first time using a sample of some 14000 QSOs. A positive detection of the Baryonic Acoustic Oscillation signature has been made at $z \simeq 2.4$ (Slosar et al. 2013), consistent with the standard $\Lambda$ CDM model. Continuing observations will tighten constraints.

An intriguing spinoff of the sample is the possibility of measuring the coherence length of structure in the IGM using phase angle differences in Fourier modes of close QSO pair spectra (Rorai, Hennawi \& White 2013). The technique promises a novel means of placing constraints on the temperature evolution and degree of clumpiness of the IGM.

Helium reionization Early numerical simulations simplified the reionization of hydrogen and helium by treating the gas as optically thin at the photoelectric edges. A correct treatment presents several modeling challenges: (1) a large simulation volume is required to capture a representative sample of QSOs, (2) radiative transfer must be included to produce the correct temperatures, (3) radiative hydrodynamical (RHD) simulations are necessary to track the photoevaporation of gas clumps overrun and heated by the advancing helium ionization fronts and (4) the mean intrinsic shape of the QSO spectra at energies exceeding the He+ photoelectric edge is not well known, and may be both luminosity and redshift dependent. While photoionization simulations in large volumes have been performed with radiative transfer in a post-processing phase (McQuinn et al. 2009; Compostella, Cantalupo \& Porciani 2014), true RHD simulations have been so far restricted to smaller volumes (Meiksin \& Tittley 2012).

Additional heating mechanisms Sources of high energy radiation, such as a hard xray background, may inject additional energy into the IGM through Compton heating (Madau \& Efstathiou 1999). Additional mechanisms include heating through plasma instabilities triggered by TeV $\gamma$-rays from blazars (Chang, Broderick \& Pfrommer 2012). No doubt additional heating sources will be suggested in the future which may affect the temperature of the IGM and constraints placed on the properties of QSO sources inferred from their contribution to heating and the shape of the UV metagalactic background radiation field.

Gas around galaxies The gaseous environment of galaxies is expected to be complex. Star formation within forming galaxies is believed to be regulated by the inflow of cold gas and the resulting feedback in the form of supernovae-driven winds or active galactic nuclei (Dekel et al. 2009). The large samples of QSOs discovered by the SDSS and the $2 \mathrm{dF}$ survey have opened up a new means of probing the gaseous environment of galaxies through the absorption signature against background QSOs (e.g., Crighton et al. 2011; Rudie et al. 2012). The larger BOSS samples will further enhance these studies. IGM simulations allowing for star formation and feedback offer a new avenue for constraining galaxy formation models (e.g., Kollmeier et al. 2006; Rakic et al. 2013; Shen et al. 2013; Meiksin, Bolton \& Tittley 2014).

Metal absorption systems The discovery of metal absorption systems in the IGM predates the identification of intergalactic Ly $\alpha$ absorption. While metals are widely regarded to have been introduced into the IGM by galaxies, possibly in winds as part of the galaxy formation process, it is unknown at what stage this happened. Even the structure of the 
metal absorbers is unclear. Photoionization modeling suggests they are co-extensive with the $\mathrm{H}$ I systems in which they are embedded on the scales of $\sim 10 \mathrm{kpc}$ (e.g., Simcoe et al. 2006), but they need not be uniformly distributed within them. They could, for instance, be transient nodules only 100 pc in size (Schaye, Carswell \& Kim 2007).

Establishing the physical state of the gas and the spectral shape and intensity of the radiation field that ionizes them is essential for inferring the metallicity of the absorbers and its evolution. Doing so would enable the metal absorbers to be used as a means for tracing the history of cosmic star formation. The spectral capacity of the TMT and the EELT will permit high precision studies of the metal systems. It will also directly reveal the presence, if any, of metals in the low density diffuse IGM for the first time, eliminating the major modeling uncertainty of radiative transfer through layers of hydrogen and helium optically thick at the photoelectric edges. They raise the possibility as well of detecting signatures of time-dependent metal ionization, placing constraints on the lifetimes or variability of QSO sources (Reynolds 2010; Oppenheimer \& Schaye 2013).

Missing physics We do not know if we have all the right physics. For example, intergalactic shocks could produce a pervasive intergalactic magnetic field. Even a weak field could alter the gas fluctuations sufficiently to have implications for the inferred ionization background required to recover the measured mean flux through the IGM (Chongchitnan \& Meiksin 2014). The cold dark matter particle may interact with itself, annihilate or decay, altering gas density profiles in collapsed haloes, including minihaloes. Possibly even general relativity must be modified on scales that would affect the large-scale distribution of matter. While current observations do not require any of these more exotic possibilities, as simulations and observations of the IGM continue to increase in precision discrepancies may find the IGM to be the first arena to offer solid evidence for the need of additional physics.

\section{References}

Bardeen, J. M., Bond, J. R., Kaiser, N., \& Szalay, A. S. 1986, ApJ, 304, 15

Blumenthal, G. R., Faber, S. M., Primack, J. R., \& Rees, M. J. 1984, Nature, 311, 517

Bond, J. R., Kofman, L., \& Pogosyan, D. 1996, Nature, 380, 603

Cen, R., Miralda-Escudé, J., Ostriker, J. P., \& Rauch, M. 1994, ApJ (Letters), 437, L9

Chang, P., Broderick, A. E., \& Pfrommer, C. 2012, ApJ, 752, 23

Chongchitnan, S. \& Meiksin, A. 2014, MNRAS, 437, 3639

Compostella, M., Cantalupo, S., \& Porciani, C. 2014, arXiv, 1407.1316

Crighton N. H. M.. et al. 2011, MNRAS, 414, 28

Croft, R. A. C., Weinberg, D. H., Katz, N., \& Hernquist, L. 1998, ApJ, 495, 44

Davé, R., Hernquist, L., Weinberg, D. H., \& Katz, N. 1997, ApJ, 477, 21

Dekel, A. et al. 2009, Nature, 457, 451

Gunn, J. E. \& Peterson, B. A. 1965, ApJ, 142, 1633

Jakobsen, P., Boksenberg, A., Deharveng, J. M., Greenfield, P., Jedrzejewski, R., \& Paresce, F. 1994, Nature, 370, 35

Jenkins, E. B. \& Ostriker, J. P. 1991, ApJ, 376, 33

Katz, N., Weinberg, D. H., Hernquist, L., \& Miralda-Escudé, J. 1996, ApJ (Letters), 457, L57

Kollmeier, J. A., Miralda-Escudé, J., Cen, R., \& Ostriker, J. P. 2006, ApJ, 638, 52

Lattanzio, J. C., Bond, J. R., \& Monaghan, J. J. 1989, BAAS, 21, 1216

Lukić, Z., Stark, C. W., Nugent, P., White, M., Meiksin, A., \& Almgren, A. 2014, ApJ (in press)

Madau, P. \& Efstathiou, G. 1999, ApJ (Letters), 517, L9

McDonald, P. et al. 2005, ApJ, 635, 761

McGill, C. 1990, MNRAS, 242, 544

McQuinn, M., Lidz, A., Zaldarriaga, M., Hernquist, L., Hopkins, P. F., Dutta, S., \& FaucherGiguère, C.-A. 2009, ApJ, 694, 842 
Meiksin, A. 1994, ApJ, 431, 109

Meiksin, A. 2009, Rev. Mod. Phys., 81, 1405

Meiksin, A., Bolton, J. S., \& Tittley, E. R. 2014, MNRAS (in press)

Meiksin, A., Bryan, G., \& Machacek, M. 2001, MNRAS, 327, 296

Meiksin, A. \& Madau, P. 1993, ApJ, 412, 34

Meiksin, A. \& Tittley, E. R. 2012, MNRAS, 423, 7

Miralda-Escudé, J. \& Rees, M. J. 1993, MNRAS, 260, 617

Miralda-Escudé, J., Cen, R., Ostriker, J. P., \& Rauch, M. 1996, ApJ, 471, 582

Oppenheimer, B. D. \& Schaye, J. 2013, MNRAS, 434, 1063

Peebles, P. J. E.. 1982, ApJ (Letters), 263, L1

Peebles, P. J. E.. 1984, ApJ, 277, 470

Peebles, P. J. E.. 1993, Principles of Physical Cosmology (Princeton, NJ: Princeton University Press)

Rakic, O., Schaye, J., Steidel, C. C., Booth, C. M., Dalla Vecchia, C., \& Rudie, G. C. 2013, MNRAS, 433, 3103

Rauch, M., Miralda-Escudé, J., Sargent, W. L. W., Barlow, T. A., Weinberg, D. H., Hernquist, L., Katz, N., Cen, R., \& Ostriker, J. P. 1997, ApJ, 489, 7

Rauch, M. \& Haehnelt, M. G. 1995, MNRAS, 275, L76

Rees, M. J. 1986, MNRAS, 218, 25P

Reynolds, S. 2010, PhD thesis, University of Edinburgh

Rorai, A., Hennawi, J. F., \& White, M. 2013, ApJ, 775, 81

Rudie, G. C. et al. 2012, ApJ, 750, 67

Sargent, W. L. W., Young, P. J., Boksenberg, A., \& Tytler, D. 1980, ApJS, 42, 41

Schaye, J., Carswell, R. F., \& Kim, T.-S. 2007, MNRAS, 379, 1169

Shen, S., Madau, P., Guedes, J., Mayer, L., Prochaska, J. X., \& Wadsley, J. 2013, ApJ, 765, 89

Simcoe, R. A., Sargent, W. L. W., Rauch, M., \& Becker, G. 2006, ApJ, 637, 648

Slosar, A., et al. 2013, JCAP, 4, 26

Smette, A., Surdej, J., Shaver, P. A., Foltz, C. B., Chaffee, F. H., Weymann, R. J., Williams, R. E., \& Magain, P. 1992, ApJ, 389, 39

Songaila, A. 2006, AJ, 131, 24

Syphers, D. \& Shull, J. M. 2014, ApJ, 784, 42

Viel, M. \& Haehnelt, M. G. 2006, MNRAS, 365, 231

Viel, M., Haehnelt, M. G., \& Springel, V. 2010, JCAP, 6, 15

Wadsley, J. W. \& Bond J. R. 1997, ASP-CS, 123, 332

Worseck, G., Prochaska, J. X., Hennawi, J. F., \& McQuinn, M. 2014, arXiv, 1405.7405

Zel'dovich, Ya. B. 1970, $A$ \& $A, 5,84$

Zhang, Y., Meiksin, A., Anninos, P., \& Norman M. L. 1998, ApJ, 495, 63

Zhang, Y., Anninos, P., \& Norman M. L. 1995, ApJ (Letters), 453, L7 